\title{
Practical Review of Peripheral Nerve Blocks in Dermatologic Surgery of the Face
}

\author{
Nicholas B. Countryman • C. William Hanke
}

Published online: 29 March 2012

(C) Springer Science+Business Media, LLC 2012

\begin{abstract}
Peripheral nerve blocks represent an invaluable tool for dermatologic surgeons. Specifically, blockade of the supraorbital and supratrochlear, infraorbital, or mental nerves provides adequate anesthesia to a large area with minimal discomfort to the patient. Extirpation of large facial carcinomas, full or partial facial resurfacing, lip augmentation, and photodynamic therapy for extensive facial actinic keratosis represent several of the commonly used applications of facial nerve blocks. Appropriate understanding of the anatomy and nuances of these peripheral nerve blocks allows the clinician to confidently perform them with minimal risks and maximum patient comfort.
\end{abstract}

Keywords Nerve $\cdot$ Blocks $\cdot$ Regional $\cdot$ Peripheral $\cdot$ Local anesthesia $\cdot$ Local anesthetic $\cdot$ Dermatology $\cdot$ Dermatologic surgery $\cdot$ Lidocaine $\cdot$ Supraorbital $\cdot$ Supratrochlear . Infraorbital $\cdot$ Mental

\section{Introduction}

The introduction of local anesthesia to the field of medicine in the early 20th century revolutionized the way in which physicians deliver care. In particular, the use of local anesthetics in dermatology and more specifically in dermatologic surgery has enabled a proliferation of procedures that can be performed in the office setting with the patient awake. Not only do local anesthetics minimize exposure to general anesthesia and the associated risks but local anesthesia also

N. B. Countryman $(\bowtie) \cdot$ C. W. Hanke

The Laser and Skin Surgery Center of Indiana,

13400 N. Meridian St., Suite 290,

Carmel, IN 46032, USA

e-mail: nicholasbcountryman@gmail.com provides a more economical method for delivering healthcare to the advantage of society as a whole. Local anesthetics can be delivered in a number of different ways including infiltrative techniques as well as topically, in nerve blocks, and in a tumescent fashion. All of these various delivery methods have various advantages and disadvantages. Dermatologists have been at the forefront of delivery of local anesthetics since the inception of the subspecialty and in particular have advanced the use of local anesthetics in regional or peripheral nerve blocks. Using this method, the local anesthetic agent is delivered into the region of the nerve trunk. Advantages of regional nerve blocks include the use of minimal local anesthetic thereby minimizing chances of toxicity and coverage of a large area without distortion of the operative site. Additionally, if performed correctly, regional nerve blocks minimize pain to the patient due to the minimal number of injections required. Numerous peripheral nerves lend themselves readily to blockade including the infraorbital, mental, supraorbital and supratrochlear, external nasal, auriculotemporal, greater auricular and transverse cervical, occipital, digital, wrist or hand, and ankle or foot blocks. The most commonly employed blocks include the infraorbital, mental, supraorbital and supratrochlear, digital, wrist or hand, and ankle or foot blocks; for the purposes of this article, the infraorbital, mental, supraorbital, and supratrochlear blocks are reviewed, specifically in relation to the practical aspects of their use in dermatologic surgery.

\section{General Principles of Peripheral Nerve Blocks}

Several general principles apply to the technique used when performing peripheral nerve blocks. A 1-inch 30-gauge needle is employed to instill the anesthetic agent into the 
space surrounding the desired nerve. Because the depth of these injections is generally deeper than that of the infiltrative technique, aspiration of the plunger of the syringe prior to injection to check for intra-vascular injection is generally recommended. Slow injection of the local anesthetic agent as with other methods minimizes the pain. Only a small amount of the anesthetic agent, generally 1 to $3 \mathrm{~mL}$, is required to achieve successful nerve blocks. The time to onset of the nerve block is approximately 5 to $10 \mathrm{~min}$ with longer time needed for large caliber nerves. Prior to initiating a procedure, assessment with perpendicular needle sticks in the entire area will ensure appropriate anesthesia. If the nerve block is incomplete, additional time may be required for large caliber nerves or repeating the block may be necessary. The duration of action depends on the agent used and whether epinephrine is added to the anesthetic. Inadvertent injection of the anesthetic agent into the nerve fibers themselves can cause significant pain as well as produce prolonged anesthesia and thus should be avoided if possible.

Amide-type anesthetics are the most commonly employed agents for regional nerve blocks. Lidocaine (Xylocaine) in a concentration of $1 \%$ is readily available and very effective for this purpose. Some recommend the use of higher concentration anesthetics such as $2 \%$ lidocaine suggesting that they provide at greater concentration gradient of anesthetic and enhance diffusion of the agent around the nerve [1]. The addition of vasoconstrictors such as epinephrine at concentrations of 1:200,000 may prolong the anesthetic effect of local anesthetics in nerve blocks. Caution should be used in digital blocks given the reports of vasospasm and distal necrosis, although this effect and risk may be exaggerated and unwarranted [2]. Use of newer anesthetic agents and proper technique obviate the need to avoid epinephrine in nerve blocks elsewhere. Plain lidocaine used in nerve blocks lasts for approximately $75 \mathrm{~min}$. Another commonly used agent, bupivacaine (Marcaine), lasts 4 to 8 times longer and is most commonly used at concentrations of $0.25 \%$ for regional nerve blocks. Generally we find that the longer-acting characteristic of bupivacaine is undesirable in most situations. A useful tool in nerve blocks is distraction [3]. Pinching, vibration, or massage of the skin adjacent to the area being blocked serve as a distraction from the pain. We find the "puppy rub" technique using ones thumb to rub the skin adjacent to the block to be the most useful, although pinching is also a simple and effective distraction technique.

\section{Peripheral Nerve Block Techniques}

The three most used nerve blocks of the face are for branches of the trigeminal nerve (cranial nerve V), which provides sensory innervation to the face. These nerves include the supraorbital and supratrochlear nerves, which are both branches of the frontal nerve that arises from the ophthalmic nerve (V1); the infraorbital nerve, which arises from the largest branch of the maxillary nerve (V2); and the mental nerve that represents a terminal branch of the mandibular nerve (V3). The areas of the face innervated by cranial nerve $\mathrm{V}$ are summarized in Table 1. The supraorbital, infraorbital, and mental nerves all exit the bone at foramina located in the mid-pupillary line (Fig. 1).

The supraorbital and supratrochlear nerves collectively supply sensation to the ipsilateral forehead and frontal scalp as well as to the vertex (Fig. 1). Blockade of these nerves can generally be accomplished with one injection. The supraorbital nerve can be located by palpating the supraorbital notch or foramen, which is readily palpable in most patients approximately $27 \mathrm{~mm}$ lateral to the glabellar midline [4]. Starting the injection in this location (Fig. 2a) within the central brow and advancing medially to the medial most

Table 1 Summary of areas innervated by cranial nerve V

\begin{tabular}{lcc}
\hline Ophthalmic nerve (V1) & Maxillary nerve (V2) & Mandibular nerve (V3) \\
\hline $\begin{array}{c}\text { Supraorbital: Anterior surface of } \\
\text { scalp and forehead; upper eyelid }\end{array}$ & $\begin{array}{c}\text { Infraorbital: Lower eyelid, lateral side wall } \\
\text { of nose, nasal alae, medial cheek, upper lip, } \\
\text { columella, anterior mucous membrane of nose, } \\
\text { and nasal spine via superior alveolar branch } \\
\text { Zygomaticotemporal: Preauricular cheek } \\
\text { and anterior temporal scalp }\end{array}$ & $\begin{array}{c}\text { Auriculotemporal: Anterior upper ear (including } \\
\text { tragus, helix, anterior half of external canal and } \\
\text { tympanic membrane) and temporal scalp. } \\
\text { Autonomic fibers to the parotid gland } \\
\text { Buccal: Buccal mucosa and cheek }\end{array}$ \\
$\begin{array}{c}\text { Supratrochlear: Anterior surface of } \\
\text { scalp and forehead, upper eyelid, } \\
\text { nasal root, bridge and upper side walls } \\
\begin{array}{c}\text { Infratrochlear: Medial upper eyelid, } \\
\text { nasal root, bridge and upper side walls }\end{array}\end{array}$ & $\begin{array}{c}\text { Zygomaticofacial: Malar region } \\
\text { Lacrimal: Conjunctive and lateral } \\
\text { upper eyelid }\end{array}$ & $\begin{array}{c}\text { Mental: Lower lip and chin } \\
\text { Anterior ethmoidal: Lower dorsum } \\
\text { and tip of nose via external nasal branch }\end{array}$ \\
\hline
\end{tabular}

(Adapted from Leonard and Hanke [7]) 


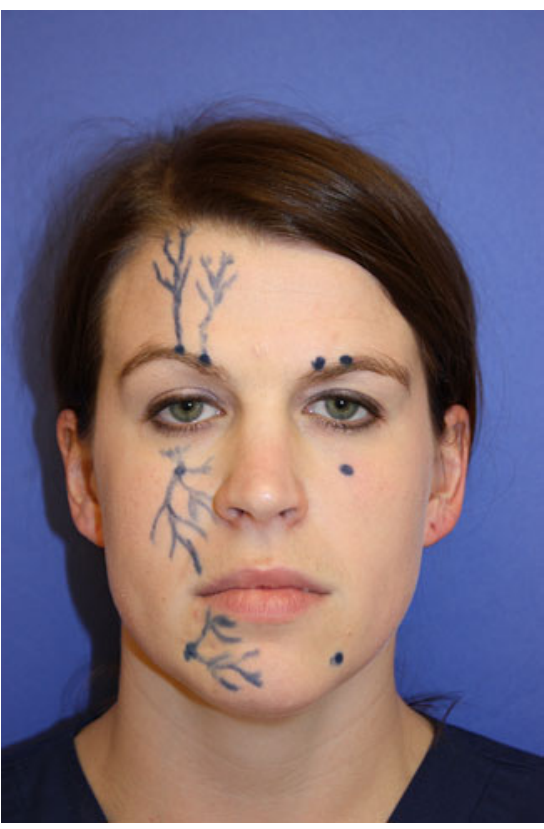

Fig. 1 Markings on the left side of the face indicate foramen in which nerves exit, with the course of the corresponding nerves marked on the right side of the model

aspect of the brow (Fig. 2b) blocks both the supraorbital and supratrochlear nerves. This often also blocks the infratrochlear nerve that innervates the medial upper eyelid, nasal root, bridge, and upper sidewalls. Approximately 2 to $4 \mathrm{~mL}$ of anesthetic is appropriate for this nerve block.

The infraorbital nerve represents the largest branch of the maxillary nerve. The infraorbital nerve block is one of the most useful nerve blocks particularly in the extirpation of large carcinomas of the ipsilateral lower eyelid, the medial cheek as well as the upper lip and the ipsilateral nasal sidewall and ala. In additional, the use of the infraorbital
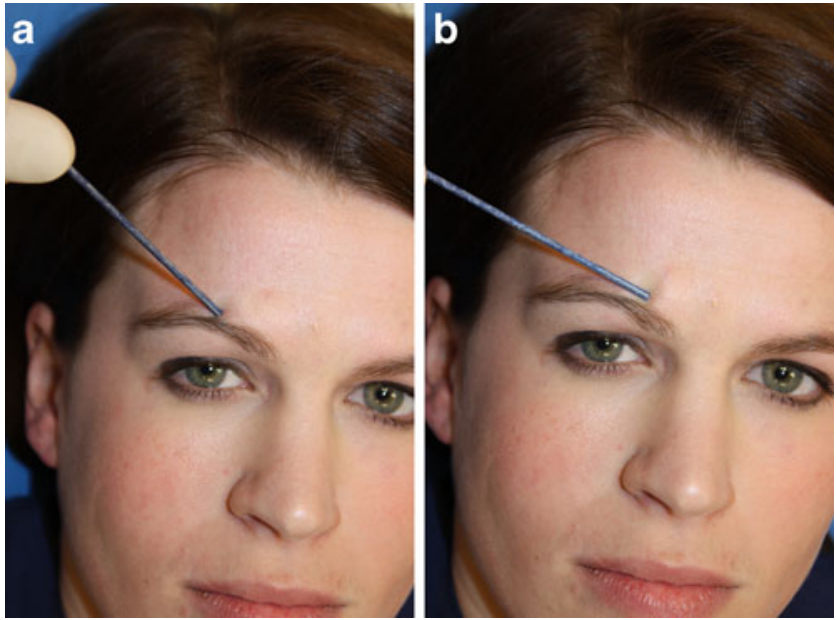

Fig. 2 Demonstration of supraorbital nerve block (panel A) and action of advancing needle (that would be subcutaneous) to achieve the supratrochlear nerve block (panel B) nerve block in conjunction with the injection of cosmetic fillers into the upper lip minimizes discomfort during these procedures. The infraorbital nerve emerges from the infraorbital foramen approximately $1 \mathrm{~cm}$ below the orbital rim at the mid-pupillary line (Fig. 1). Two approaches exist to access this foramen for blockade: the intraoral route and the percutaneous route. In the intraoral route, a small amount of a topical anesthetic gel such as lidocaine or benzocaine on a cotton-tipped applicator is used for topical anesthesia. Placement of cotton-tipped applicator at the root of the canine tooth in the gingival-buccal sulcus for 1 to 5 min depending on the agent used eases the insertion of the needle into the mucosa. Placing the pointer finger of the non-dominant hand over the infraorbital foramen serves as a "target" when advancing the needle. The needle is introduced at the anesthetized area in the gingival-buccal sulcus at the space between the second and third teeth from the midline. The needle is then advanced towards the nondominant pointer finger at the foramen (Fig. 3a). Repeating this process but introducing the needle at the space between the third and fourth teeth from midline will ensure complete blockade (Fig. 3b). Light palpation with the pointer finger serves as a guide in determining if the tip of the needle is in the appropriate location. Approximately $2 \mathrm{~mL}$ of anesthetic is generally sufficient to anesthetize the infraorbital nerve. An additional $0.5 \mathrm{~mL}$ of anesthetic injected into the maxillary phrenulum may be necessary to provide complete block of the upper lips (Fig. 3c). In the percutaneous approach, the needle can be introduced in a perpendicular fashion to the skin approximately $1 \mathrm{~cm}$ below the orbital rim at the mid-pupillary line down to the bone, withdrawing a tenth of a millimeter until approximately $2 \mathrm{~mL}$ of anesthetic is injected (Fig. 4). This can be followed by injection of another 1 to $2 \mathrm{~mL}$ of anesthetic inferior medially (Fig. 4b) and inferior laterally (Fig. 4c) to obtain an optimal infraorbital nerve block.

Mental nerve blocks are particularly useful not only in the extirpation of large carcinomas of the lower lip but also cosmetic procedures such as lip augmentation with hyaluronic acid fillers. Successful blockade of the mental nerve provides anesthesia to the skin and mucosa of the lower lip and chin. Again two viable methods exist for this nerve block: the intraoral route and the percutaneous route. As with the infraorbital block, the intraoral approach is generally preferred due to patient comfort and ease of achieving a successful block. Similar to the intraoral approach in blockade of the infraorbital nerve, use of a small amount of a topical anesthetic gel at the root of the canine tooth at the gingival-buccal sulcus for 1 to $5 \mathrm{~min}$ depending on the agent used can ease the insertion of the needle into the mucosa. The needle is introduced at the anesthetized area in the gingival-buccal sulcus at the space between the third and fourth teeth from the midline and is advanced towards the 
Fig. 3 Demonstration of the intraoral approach to the infraorbital nerve block. Initially the needle is inserted and advanced towards the infraorbital foramen at the gingival-buccal sulcus in the space between the 2 nd and $3 \mathrm{rd}$ teeth lateral to midline (panel A). This is repeated at the space between the $3 \mathrm{rd}$ and 4 th teeth (panel B) to achieve a more complete block. Finally, the injection into the mandibular frenulum (panel $C$ ) completes the block of the upper lip
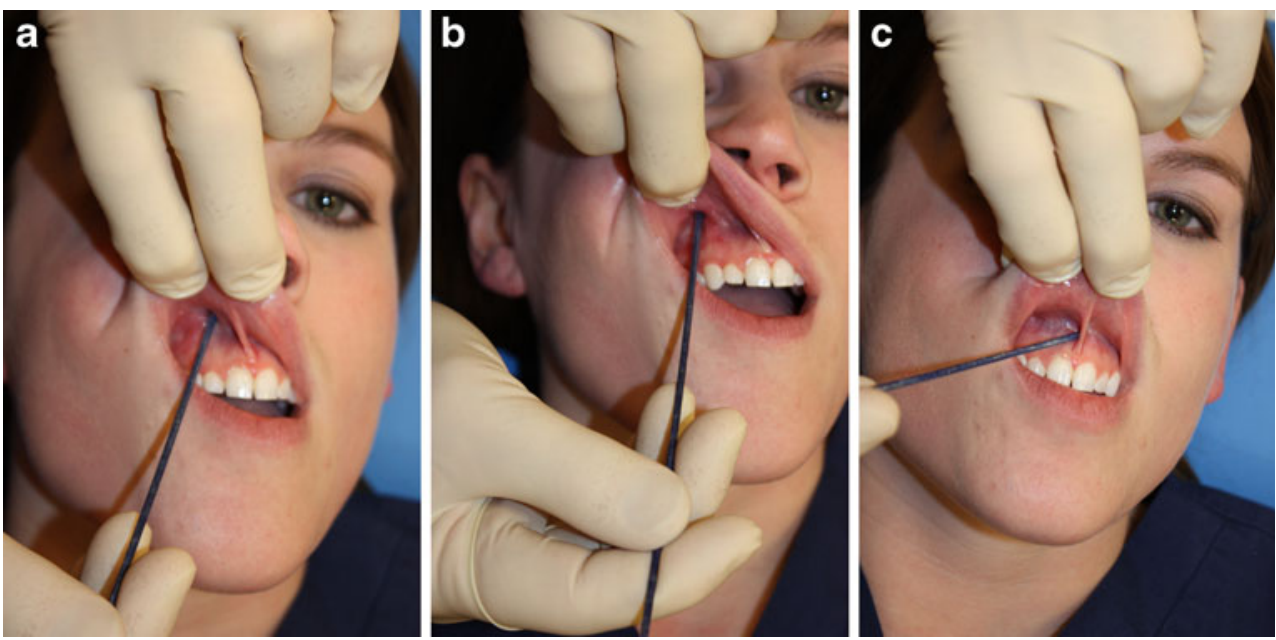

foramen (Fig. 5a). Repeating this process but introducing the needle at the space between the fourth and fifth teeth from midline will ensure a more complete blockade (Fig. 5b). An additional $0.5 \mathrm{~mL}$ of anesthetic injected into the mandibular frenulum may be necessary to provide complete block of the lower lips (Fig. 5c). In the percutaneous approach, the needle can be introduced in a perpendicular fashion to the skin approximately $1 \mathrm{~cm}$ above the lower mandible at the midpupillary line down to the bone, withdrawing $1 / 10$ th of a millimeter until approximately $2 \mathrm{~mL}$ of anesthetic is injected (Fig. 6a). This can be followed by injection of another 1 to $2 \mathrm{~mL}$ of anesthetic in a slight superior-medial direction (Fig. 6b) and inferior-medially (Fig. 6c) to obtain an optimal infraorbital nerve block.

\section{The "Mucosal" Nerve Block}

"Mucosal" mental and infraorbital "peripheral" nerve blocks are similar to the mental and infraorbital nerve blocks. A small depot of anesthetic can be infiltrated superficially in the mucosa at similar entry points as the mental and infraorbital nerve blocks causing the mucosa to blanch. As previously discussed, an additional $0.5 \mathrm{~mL}$ of anesthetic injected into the frenulum may be necessary to provide complete block of the upper and lower lips. Though not a true peripheral nerve block, this method provides adequate anesthesia to a more focal area. This eliminates one of the patient's common complaints of the large anesthetized area for cosmetic injection into the lip. A similar technique has been described in which several infiltrations are spaced along the mandibular or maxillary vestibule in order to achieve upper or lower lip anesthesia [5]. Similarly, this may be used in association with cryosurgery or $\mathrm{CO}_{2}$ laser destruction of extensive actinic cheilitis.

\section{Potential Complications, Risks, and Disadvantages of Nerve Blocks}

Complications encountered during nerve blocks are exceedingly rare and almost universally reversible. The most
Fig. 4 Demonstration of the percutaneous approach to the infraorbital nerve block. Initially the needle is inserted perpendicular to the foramen (panel $A$ ), then advanced inferior medially (panel $B$ ) and inferior laterally (panel $C$ ) to achieve a more complete block by injecting around the major branches of the infraorbital nerve (refer to Fig. 1 for illustration)
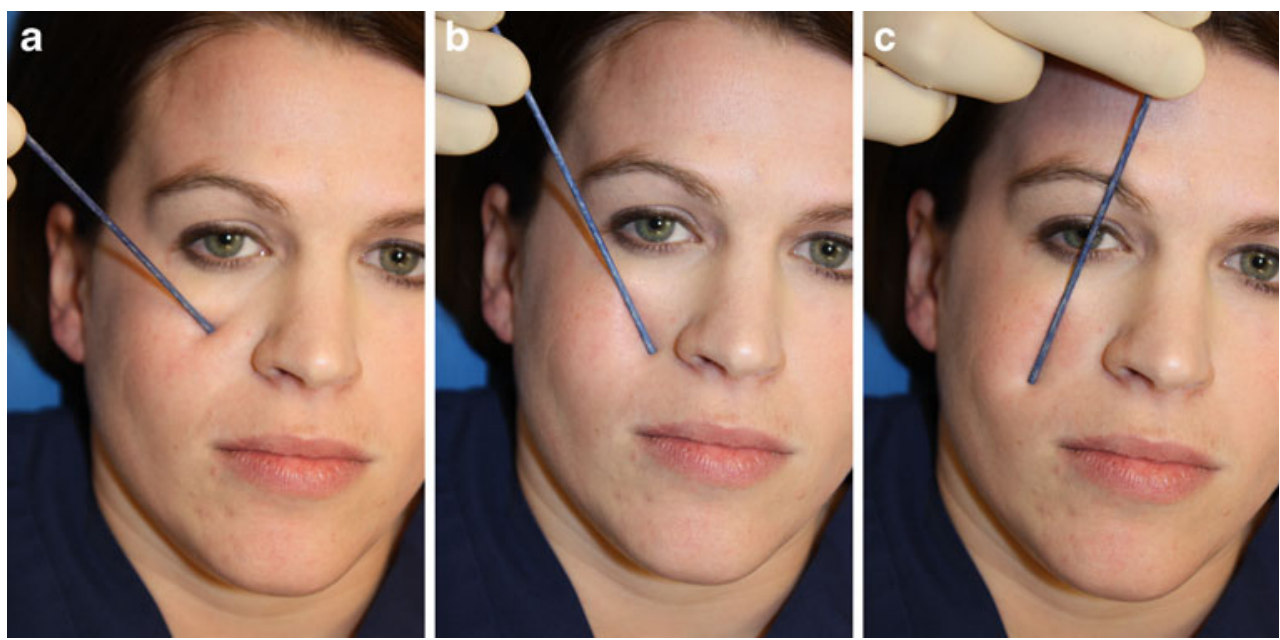
Fig. 5 Demonstration of the intraoral approach to the mental nerve block. Initially the needle is inserted and advanced towards the mental foramen at the gingival-buccal sulcus in the space between the $3 \mathrm{rd}$ and 4th teeth lateral to midline (panel $A$ ). This is repeated at the space between the 4 th and 5 th teeth (panel B) to achieve a more complete block. Finally, the injection into the maxillary frenulum (panel C) completes the block of the lower lip
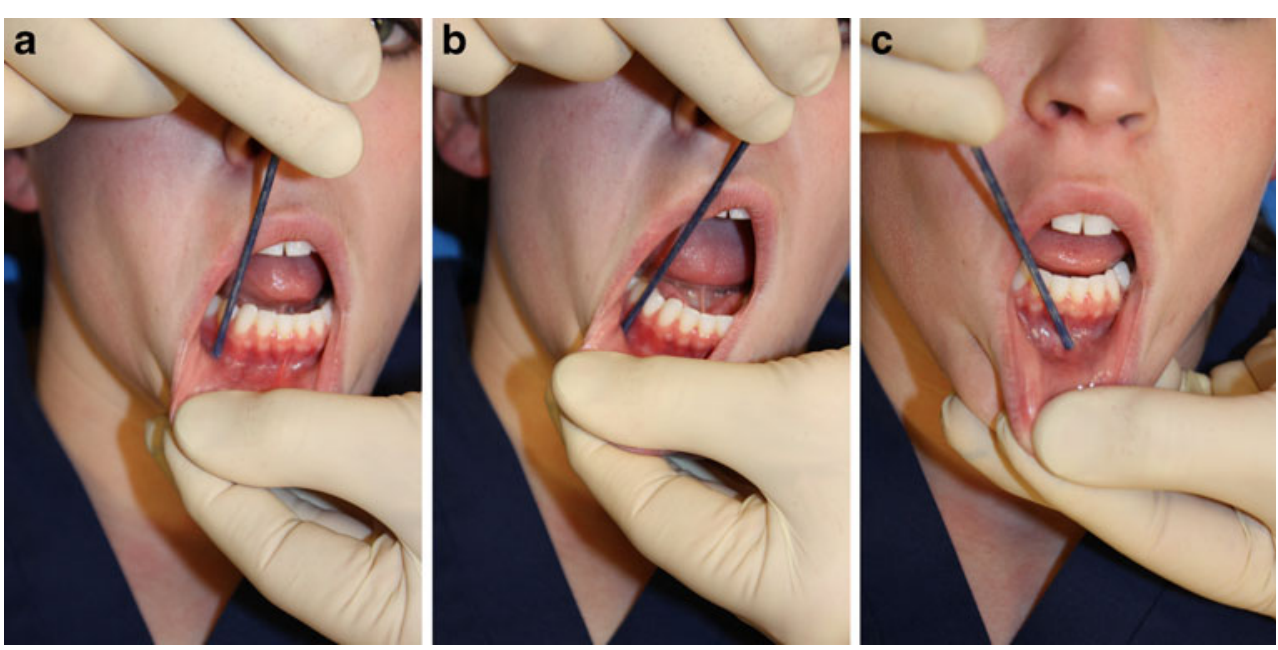

reported complications include intravascular injection, vessel trauma causing ecchymoses and hematoma, or nerve injury leading to dysesthesias and paresis. Proper technique and withdrawing the plunger prior to injection of the anesthetic agent minimizes the risk of intravascular injection as well as nerve injury. If sudden radiating pain is encountered in the area during advancement of the needle for the nerve block, this likely represents direct contact between the needle and the nerve, which can lead to nerve injury. The needle should be withdrawn and redirected. The anesthetic agent should not be injected as this risks injection immediately into the nerve. The infiltration method of local anesthesia is generally performed with the addition of epinephrine and thus has the advantage that this method leads to vasoconstriction of the adjacent blood vessels. This in turn leads to decreased bleeding at the operative site. Nerve blocks lack this effect and may require additional local anesthetic with epinephrine be infiltrated in the operative site to help achieve hemostasis.

Fig. 6 Demonstration of the percutaneous approach to the mental nerve block. Initially the needle is inserted perpendicular to the foramen (panel $A$ ), then advanced superior medially (panel B) and inferior medially (panel $C$ ) to achieve a more complete block by injecting around the major branches of the mental nerve (refer to Fig. 1 for illustration)
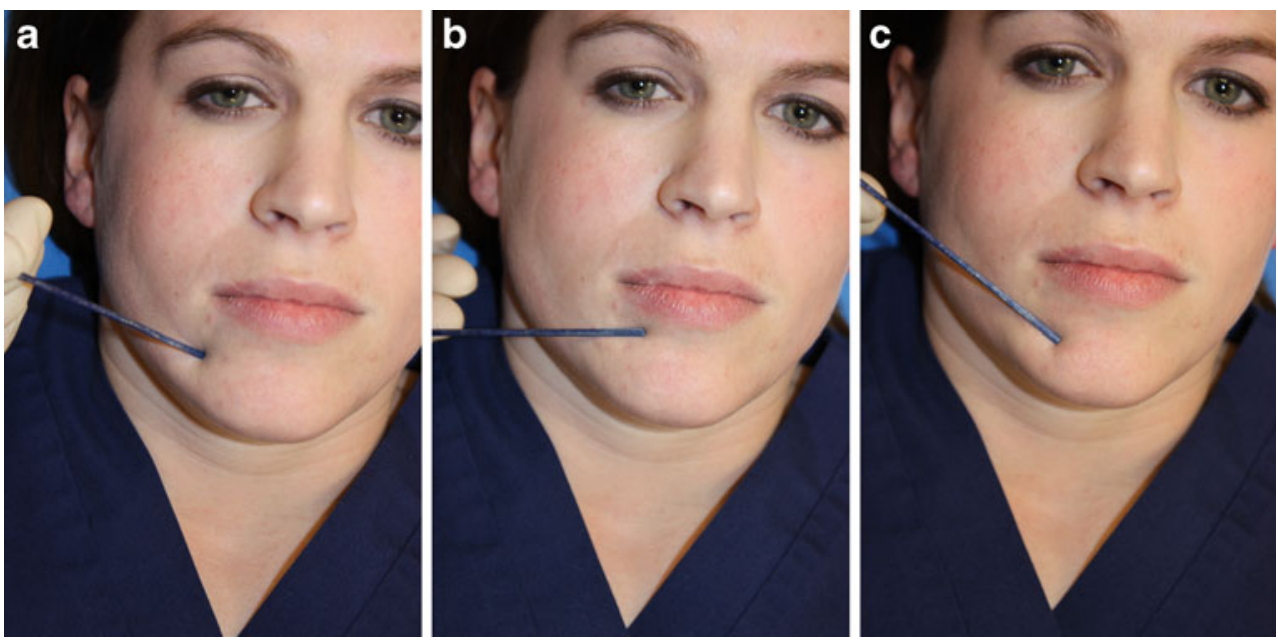

\section{Which Block to Choose for a Specific Application?}

Nerve blocks have been successfully utilized for a variety of applications. Nerve blocks anesthetize a large area with minimal anesthetic agent. Thus, for extirpation of large facial carcinomas, supraorbital and supratrochlear, infraorbital, or mental nerve blocks can be utilized alone or in conjunction with infiltrative techniques to maximize patient comfort and minimize the amount of anesthetic needed. Full-face dermabrasion as well as deep full-face resurfacing can be successfully and nearly painlessly undertaken using peripheral nerve blocks of the supraorbital and supratrochlear, infraorbital, or mental nerve. Additional, bilateral infraorbital blocks with or without the addition of an injection of $0.5 \mathrm{~mL}$ of anesthetic into the maxillary labial frenulum provides adequate anesthesia for upper lip dermabrasion for deeper perioral rhytids. The addition of bilateral maxillary nerve blocks with injection into the mandibular labial frenulum to the previous block is useful for cosmetic lip augmentation. Alternatively, the "mucosal" block of the upper and lower lips negates the need for the total 
nerve block and is generally found to be preferred by patients. Facial nerve blocks of cranial nerve $\mathrm{V}$ have been shown to improve patient comfort during photodynamic therapy of extensive facial actinic keratosis [6].

\section{Conclusions}

With appropriate technique and practical tips reviewed here, successful peripheral nerve blocks of the infraorbital, mental, supraorbital, and supratrochlear nerves can be achieved. Use of these blocks for specific applications as previously outlined improves patient and thus physician comfort. This patient comfort improves patient satisfaction with the procedure being performed.

Disclosure No conflicts of interest relevant to this article were reported.

\section{References}

1. Dinehart SM. Topical, local, and regional anesthesia. In: Wheeland R, editor. Cutaneous surgery. Philadelphia: WB Saunders; 1994. p. 109.

2. Krunic AL, et al. Diginal anesthesia with epinephrine: an old myth revisited. J Am Acad Dermtol. 2004;51(5):755-9.

3. Eaton JS, Grekin RC. Regional anesthesia of the face. Dermatol Surg. 2001;27:1006.

4. Niamtu J. Local anesthetic blocks of the head and neck for cosmetic facial surgery, II: techniques for the upper and mid face. Cosmetic Surg. 2004;17:584-5.

5. Niamtu, J. Local anesthetic blocks of the head and neck for cosmetic facial surgery, IV: techniques for the upper and mid face. Cosmetic Surg. 2004;17:714-20.

6. Paoli J, et al. Nerve blocks provide effective pain relief during topical photodynamic therapy for extensive facial actinic keratosis. Clin Exp Dermatol. 2008;33(5):559-64.

7. Leonard AL, Hanke CW, editors. Cosmetic dermatology procedure manual for dermatology residents and practicing dermatologist. 2007. 